\title{
The Design and Construction of LHCb VELO Upgrade Modules
}

DOI:

10.1109/NSS/MIC42101.2019.9059693

\section{Document Version}

Accepted author manuscript

Link to publication record in Manchester Research Explorer

\section{Citation for published version (APA):}

Svihra, P. (2020). The Design and Construction of LHCb VELO Upgrade Modules. In 2019 IEEE Nuclear Science Symposium and Medical Imaging Conference, NSS/MIC 2019 [9059693] (2019 IEEE Nuclear Science Symposium and Medical Imaging Conference, NSS/MIC 2019). IEEE. https://doi.org/10.1109/NSS/MIC42101.2019.9059693

\section{Published in:}

2019 IEEE Nuclear Science Symposium and Medical Imaging Conference, NSS/MIC 2019

\section{Citing this paper}

Please note that where the full-text provided on Manchester Research Explorer is the Author Accepted Manuscript or Proof version this may differ from the final Published version. If citing, it is advised that you check and use the publisher's definitive version.

\section{General rights}

Copyright and moral rights for the publications made accessible in the Research Explorer are retained by the authors and/or other copyright owners and it is a condition of accessing publications that users recognise and abide by the legal requirements associated with these rights.

\section{Takedown policy}

If you believe that this document breaches copyright please refer to the University of Manchester's Takedown Procedures [http://man.ac.uk/04Y6Bo] or contact uml.scholarlycommunications@manchester.ac.uk providing relevant details, so we can investigate your claim.

\section{OPEN ACCESS}




\title{
The Design and Construction of LHCb VELO upgrade modules
}

\author{
Peter Svihra* on behalf of $\mathrm{LHCb}$
}

\begin{abstract}
* Department of Physics and Astronomy, School of Natural Sciences, University of Manchester, M13 9PL, Manchester, UK peter.svihra@cern.ch
\end{abstract}

\begin{abstract}
The construction of the new LHCb Vertex Locator (VELO) detector is presented. The upgraded subsystem will play a crucial role in the tracking during data-taking runs starting in 2021, its main objective locating primary and secondary vertices. Compared to its predecessor, the main advantages are better resolution together with trigger-less readout at the maximal rate of $40 \mathrm{MHz}$. In total, VELO consists of 52 modules positioned in vacuum along the LHC beampipe, surrounding the interaction point. The modules are populated with 4 hybrid silicon pixel detectors with pixel pitch of $55 \mu \mathrm{m}$. Each of the sensors is read out by 3 VeloPix ASICs with 256x256 pixels. For experiment control and data propagation, sets of front-end hybrids and GBTx ASICs are utilized. The data are then sent through a vacuum feed-through board to an opto-and-power (OPB) board, which is connected to the rest of the experiment via optical fibres. Cooling of the whole module is achieved by phase transition of liquid $\mathrm{CO}_{2}$ using a custom-made silicon micro-channel substrate.

The assembly of modules at both University of Manchester (Manchester, UK) and Nikhef (Amsterdam, NL) requires high precision in many aspects, therefore extensive procedures for the large-scale construction and its quality assurance have been deployed. The information during each step is uploaded to the online database and automatically analyzed, providing instantaneous information about quality of both components, performed tasks and whole modules. Final assembly of the whole system then takes place at University of Liverpool (Liverpool, UK) and is then transported to CERN (Geneva, CH).

Index Terms-LHC, hybrid pixel detectors, radiation resistant, tracking systems
\end{abstract}

\section{INTRODUCTION}

$\mathrm{LHCb}$ is a complex detector system operated at one of the interaction points of LHC at CERN. The detector is a single arm forward spectrometer, its main purpose is to study $\mathrm{CP}$ violation, rare decays and more, focusing on b- and c- hadron decays [1]. The schematic layout of the LHCb is in figure 1.

After a successful 10 year-long operation and delivered integrated luminosity of $10 \mathrm{fb}^{-1}$, the system is being upgraded with novel subdetectors. The upgrade is occuring during second Long Shutdown over a period of two years 2019-2020 with commissioning during first half of the year 2021.

The VELO (VErtex LOcator) is its crucial system used for tracking and locating vertices, the original system consisted of silicon strip detectors, arranged to determine $r$ and $\phi$ coordinates of the passing particles. It was the system closest to, and surrounding, the interaction point [3], [4]. The new system consists of silicon pixel detectors, increasing the effective number of channels from 180k up to almost $41 \mathrm{M}$.

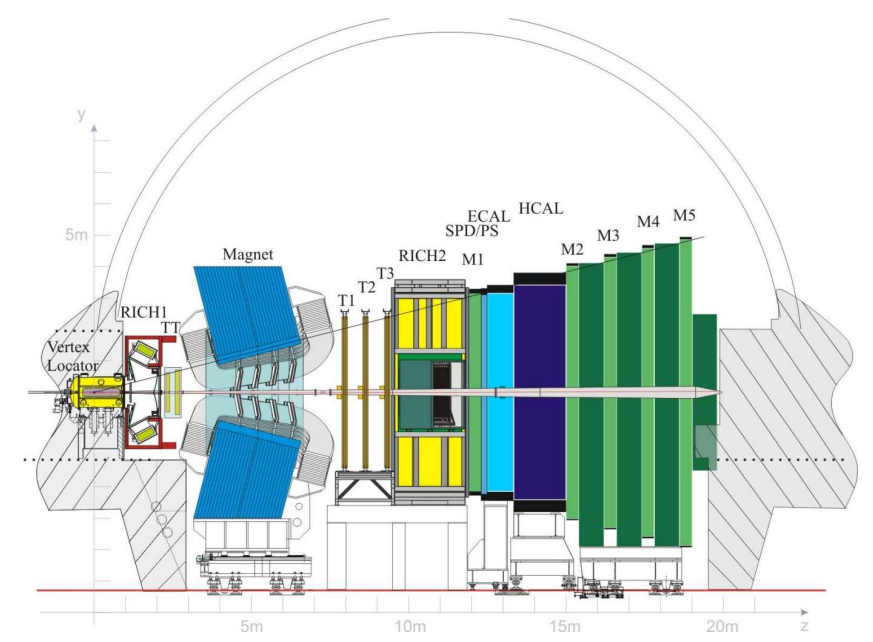

Fig. 1: Schematic view of the LHCb experiment [2].
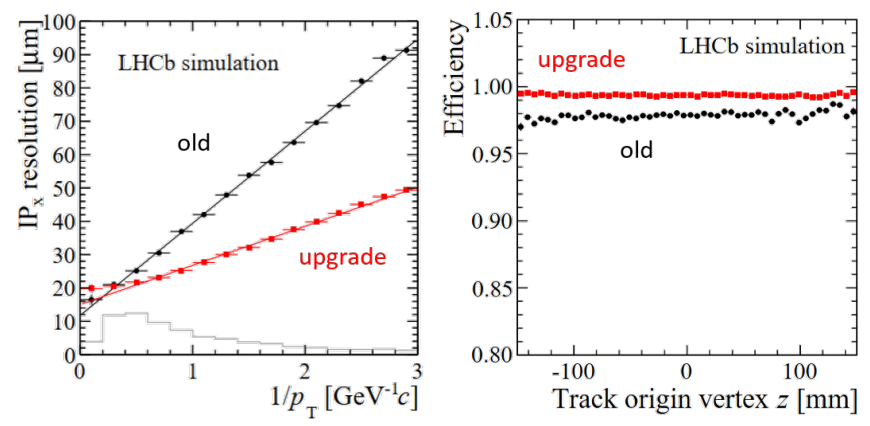

Fig. 2: Comparison of simulations focusing on impact parameter resolution (left) and efficiency (right) of old and upgraded VELO [2].

In addition, whereas the previous VELO was triggered with $1 \mathrm{MHz}$ readout, the new system is readout untriggered at the beam-crossing frequency of $40 \mathrm{MHz}$ [2]. Simulations of both resolution and efficiency for old and upgrade VELO are in figure 2 .

\section{Module DesigN}

In total, the VELO upgrade consists of 52 modules positioned in vacuum along the LHC beampipe, surrounding the interaction point [5]. For the best tracking performance the smallest possible amount of material has to be used in con- 


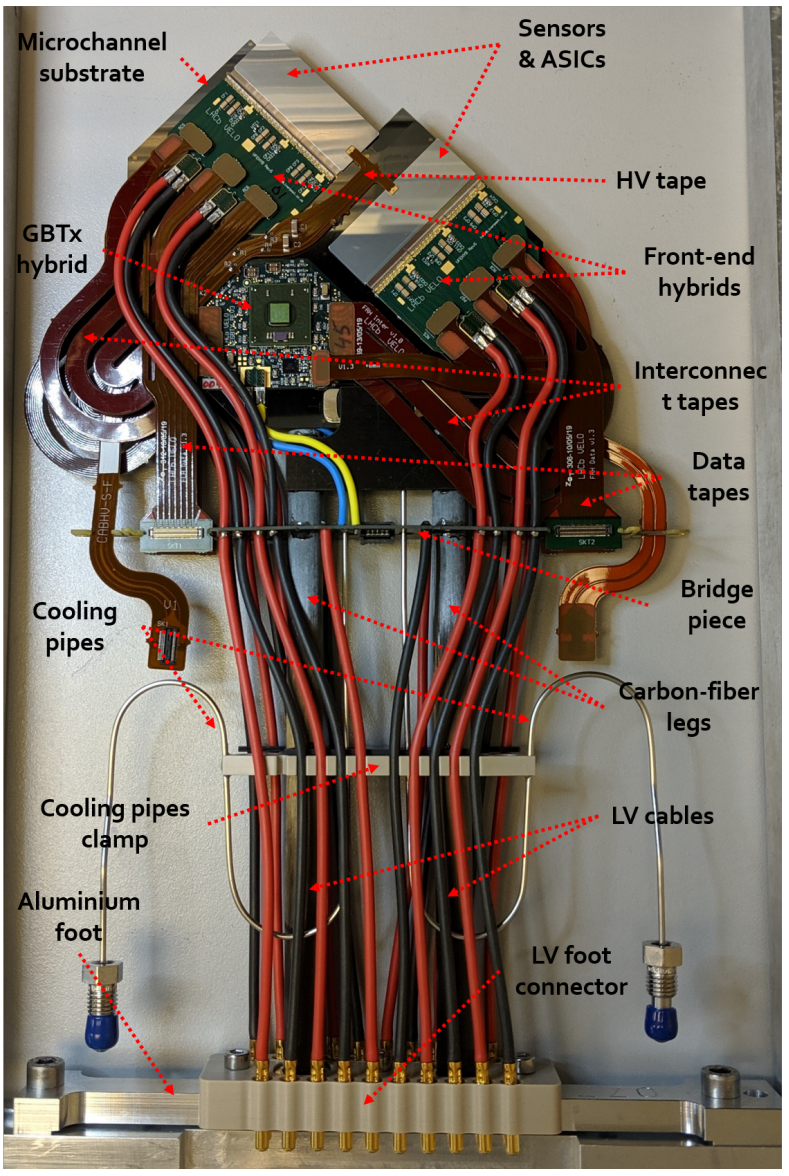

Fig. 3: Finished VELO upgrade module.

structing all components. This also helps lowering radiation damage of the sensitive parts.

The VELO upgrade modules are populated with 4 hybrid silicon pixel detectors with pixel pitch of $55 \mu \mathrm{m}$. Each of the sensors is read out by 3 VeloPix ASICs with 256x256 pixels [6]. HV for biasing is delivered through thin HV tapes to sensors, operated up to maximum of $1000 \mathrm{~V}$.

For experiment control and data propagation, sets of frontend hybrids and GBTx ASICs are utilized. The data are then sent through a vacuum feed-through board to an OPB (Optoand-Power Board), which is connected to the rest of the experiment via optical fibres. Cooling of the whole module is achieved by evaporation of liquid $\mathrm{CO}_{2}$ operated at $-30{ }^{\circ} \mathrm{C}$, using a custom-made silicon micro-channel substrate [7].

In order to operate the detector in vacuum conditions close to the interaction point without possible contamination of primary beampipe vacuum, a thin aluminium RF foil is used for vacuum separation and protection from beam-induced effects [2].

An example of a final design of a fully assembled module is in figure 3.

\section{Module CONSTRUCTION}

The assembly of the modules consists of many different steps, requiring high precision at each one. At first, the bare

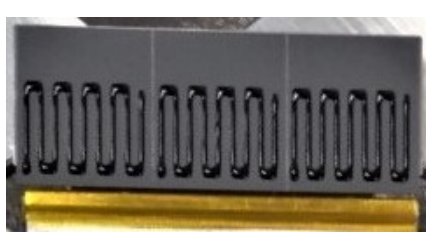

(a) Snake pattern.

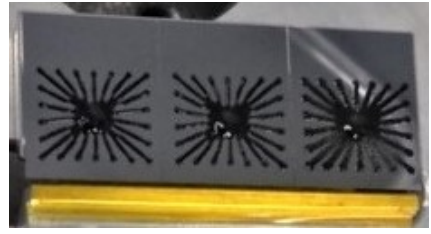

(b) Star pattern.
Fig. 4: Comparison of alternative glue patterns deposited on tiles. The star pattern (right), due to it's better properties, was selected for the module construction.

module consisting of aluminium foot, carbon fibre legs, midplate and the cooling substrate is constructed. Afterwards, tiles and hybrid ASICs are glued, wire-bonded and the rest of the flat cables for communication and $\mathrm{HV}$ are attached.

A more detailed description of some of the most critical assembly steps follows.

\section{A. Tiles Alignment}

Placement of the tiles on the modules is one of the most precise tasks during module construction, aiming at achieving a precision better than $30 \mu \mathrm{m}$. Alignment is done using custom-built mechanical jigs, a reference frame is measured using two precision markers known with respect to the module foot.

Each tile is placed on a dedicated vacuum mount equipped with 2 independent translation stages and a rotation stage. A camera mounted on the motion system allows for pattern recognition and position measurement of the alignment markers etched on the ASICs during fabrication.

\section{B. Gluing}

For the best cooling performance delivered to all VeloPixes, a thin uniform layer of glue has to be deposited, Stycast 2850FT with catalyst 9 were selected for this purpose [8]. The hybrid ASICs attached using a standard loctite glue, HV tapes using a drop of Araldite 2020 and wire-bonding.

The aligned tiles are transferred using a vacuum holder to the gluing robot, the glue deposition is then performed automatically.

The glue layer has to not only be uniform at around $80 \mu \mathrm{m}$ but also trapping of air has to be avoided, as the expansion of the trapped air pockets during the detector operation in vacuum could cause damage. For this purposes, star shaped pattern has been tested and selected. Compared to other options (e.g. a snake-like pattern), there is a smaller chance to trap air or spill glue over the edge due to the smaller deposited amount in those regions, without reducing the overall coverage of the ASIC. The comparison of two patterns deposited is in figure 4.

\section{Wire-bonding}

Each of the 4 tiles must be wire bonded to its associated hybrid circuit, with a total of 420 bonds per tile. The procedure is done separately for the two sides, connection of ASICs to 

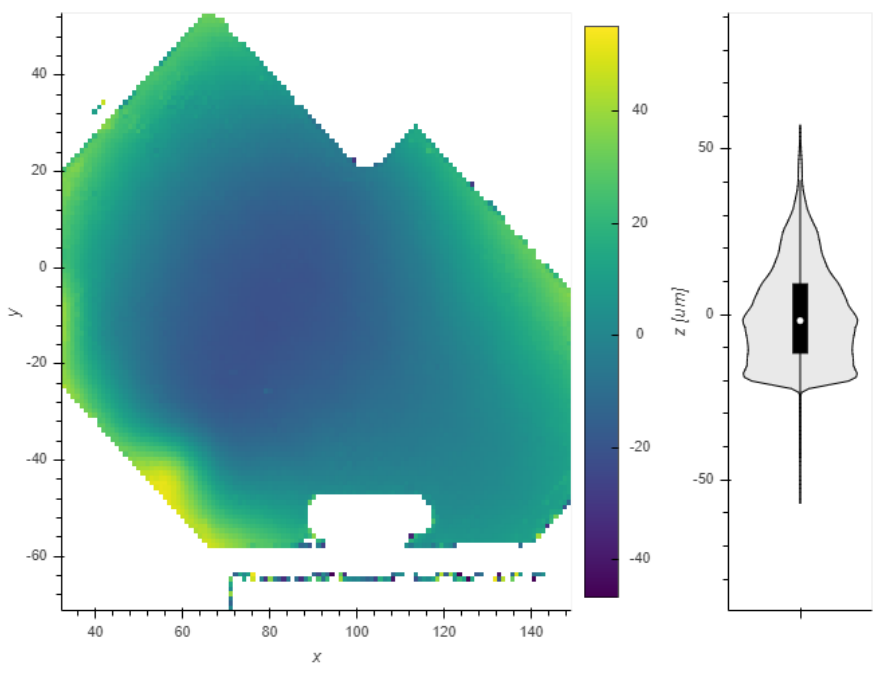

(a) Measured at CERN.
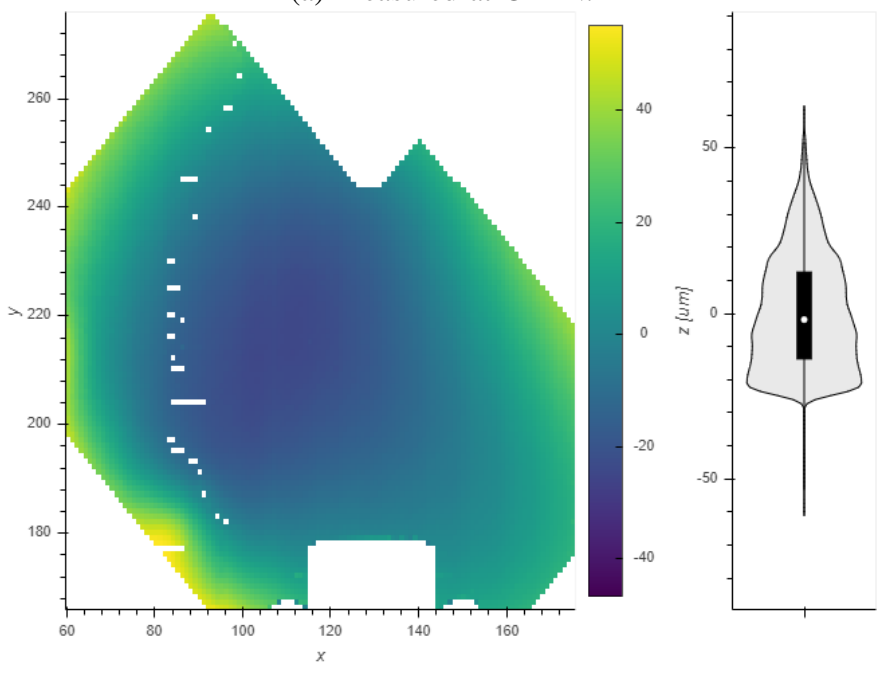

(b) Measured at Manchester.

Fig. 5: Measurement of flatness of the cooling substrate. Visualized as a heat map (left) and a violin-plot (right) of the $\Delta z$ value comparing the fitted plane with raw data.

the front-end hybrids and HV tapes to the sensor surface in one go.

\section{QuAlity Assurance}

Every component is graded when produced, however, the final quality of the module might be lowered by an imprecision during any of the assembly steps. Because of that, a variety of checks are performed over the course of the assembly, split into three categories: metrology (measurements of building precision), mechanical performance (effects of high voltage and cooling) and communication (data acquisition chain tests). Each test is then assigned a grade from a list: A - production grade; B - insignificant issues; C - moderate issues; F - QA failure.

The same set of tests is done at both assembly sites (Manchester and Nikhef) and CERN, differing only in some
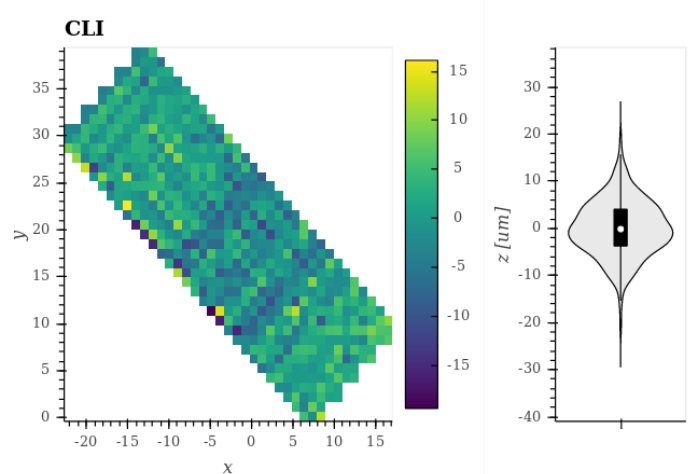

(a) Tile flatness.
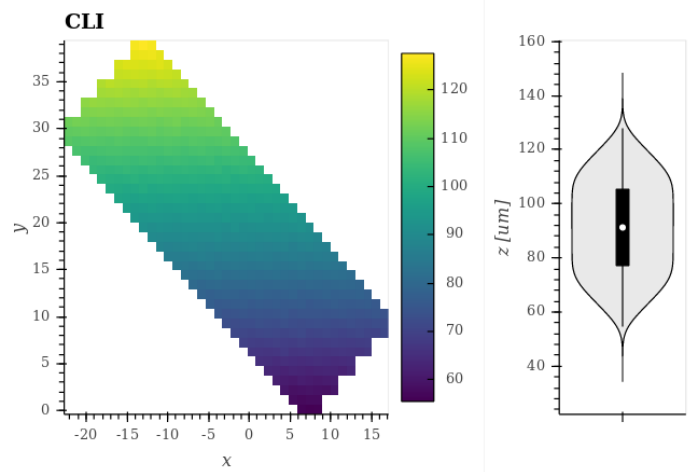

(b) Glue layer thickness.

Fig. 6: Measurement of tile flatness and glue-layer thickness. Visualized as a heat map (left) and a violin-plot (right) of the $\Delta z$ value comparing the fitted plane with raw data.

of the tools and data formats used. Some of the modules have been tested at all three places to cross-verify the setups and minimize the errors from production.

\section{A. Metrology}

1) Substrate flatness: Flatness measurement of the cooling substrate is performed in order to check the quality and spot any damage that could have happened during production, transport or storage of the substrates. It is performed both at the cooling substrate production site (CERN) and at each of the assembly sites.

Different tools are used for the measurement, relying on optical or laser systems. Values of $x, y$ and $z$ are obtained, $\Delta z$ is then obtained by plane fitting.

A comparison between measurement performed at CERN and at Manchester is in figure 5. The flatness measurements are in correlation (as seen from the heatmap and violin plot distribution). Both measurements indicate a peak in bottom left corner, corresponding to the known crack in the micro-channel plate for that particular low-grade substrate.

Grading of the measurement is defined using $\eta_{25}$ and $\eta_{75}$ of $\Delta z$ values, results being A $( \pm 50 \mu \mathrm{m}), \mathrm{B}( \pm 75 \mu \mathrm{m}), \mathrm{C}$ $( \pm 100 \mu \mathrm{m})$ and $\mathrm{F}$ (outside).

2) Glue layer thickness and tile flatness: Measurement of the glue layer thickness underneath the tiles is performed in 

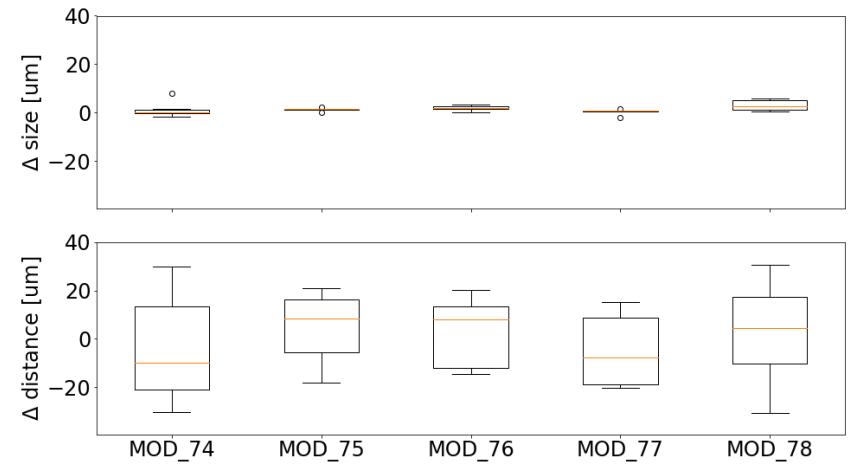

Fig. 7: Measured distances of the sensor markers. Top plot shows comparison between ideal and measured distance of each two markers on a single tile (systematic error of measurement), bottom plot shows comparison between ideal and measured distance of each point to the $\mathrm{LHCb}(0,0)$ coordinate (misalignment of the tiles).

order to check the quality of the deposition process and for later comparison with the module thermal performance. As a by-product of this test, the flatness of the tiles is also measured.

Measurement of the glue is similar to the substrate flatness, the thickness is obtained through a two-step fitting of both sensor surface and the surrounding substrate area. Resulting glue thickness is estimated as a difference between the two, taking the thickness of the tiles of $445 \mu \mathrm{m}$ into consideration.

Results from one of the measurements are in figure 6. The non-uniformity of the glue layer is caused by the difference between the planes of the micro-channel substrate and the tile, the gradient pointing in the outward direction from the centre of the substrate corresponds to the profile measured during the substrate flatness.

Grading of both tile flatness and glue layer thickness is done by comparing $\mu \pm 2 \cdot \sigma$ with selected ranges. For the tile flatness the ranges are defined as $\mathrm{A}( \pm 20 \mu \mathrm{m}), \mathrm{B}( \pm 40 \mu \mathrm{m})$, $\mathrm{C}( \pm 60 \mu \mathrm{m})$ and $\mathrm{F}$ (outside). The glue thickness ranges are $\mathrm{A}$ (from $40 \mu \mathrm{m}$ to $120 \mu \mathrm{m}$ ), B (from $30 \mu \mathrm{m}$ to $150 \mu \mathrm{m}$ ), C (from $20 \mu \mathrm{m}$ to $180 \mu \mathrm{m}$ ) and $\mathrm{F}$ (outside).

3) Tile position: The exact position of all tiles is crucial for a better starting point of the alignment in the experiment. The measurements are performed using the markers on ASICs and on the back side of the sensor, for the tiles which overhang the cooling substrate. This enables comparison of both sides with respect to each other.

Results of the measurement compared to the ideal position from the design are plotted in figure 7. Comparing distances of the pairs of markers on the same tile enables estimation of the device measurement error.

Grading of the test is done using both absolute values of differences between measured and ideal position $\Delta x$ and $\Delta y$, A $(<30 \mu \mathrm{m}), \mathrm{B}(<45 \mu \mathrm{m}), \mathrm{C}(<60 \mu \mathrm{m}), \mathrm{F}$ (outside).

4) Pull test: To check the quality of wire-bonding, each ASIC contains 4 bonds which are not electric connections.

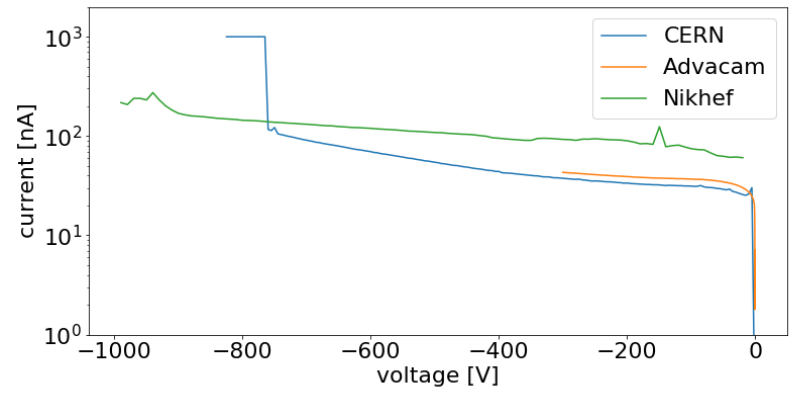

Fig. 8: IV measurement of the same sensor at different sites and stages of the production.

Half of the sacrificial bonds are pulled and breaking forces recorded before finishing the module, the other half is pulled after all testing steps, before shipping to the VELO assembly site.

Grading of the test is defined by breaking force of the bonds in grams, A (all $>5 \mathrm{~g}$ ), B (any from $2 \mathrm{~g}$ to $5 \mathrm{~g}$ ), C (any $<2 \mathrm{~g}$ ), $\mathrm{F}$ (multiple failed).

\section{B. Mechanical performance}

1) IV curves: As the simplest way to check the quality of a known silicon sensor, IV curves of all modules are taken at different steps of production. The IV measurement always contains a level of variation due to the environment therefore the results are only monitored in order to spot large defects in performance.

Comparison of data taken after bump-bonding at Advacam, probe-station at CERN and of the full sytem with assembled module at Nikhef is in figure 8 .

2) Displacement: During installation, evacuation and cooling of the module, the position of the module can change in the $z$ beam direction. The displacement is caused by different thermal coefficients or stress of the connecting materials.

Understanding this shift is crucial as it can affect the performance of reconstructing the particle tracks in the experiment. The final position of the modules will be measured during assembly of the whole VELO at Liverpool, however, the displacement of every module is checked for each step of the installation. This is needed to make sure the modules will not deform too much or get close to the RF foil on either side.

Displacement is measured using capacitive sensors at the tip of the tiles and together with the temperature of the liquid $\mathrm{CO}_{2}$ monitored as a function of time. Results are in figure 9. These measurements show maximal variation of around $80 \mu \mathrm{m}$ at the tip of the module, consistent for all built modules and even during multiple and independent cooling-down cycles.

Grading is defined according to the maximal displacement of any of the sensors, A $(<100 \mu \mathrm{m}), \mathrm{B}(<150 \mu \mathrm{m}), \mathrm{C}(<$ $200 \mu \mathrm{m}), \mathrm{F}$ (outside).

3) Thermal cycling: To spot any early deaths of the modules at the experiment, a thermal cycling of 10 cycles is performed, ranging from $-30^{\circ} \mathrm{C}$ to $20^{\circ} \mathrm{C}$. At the end, a 


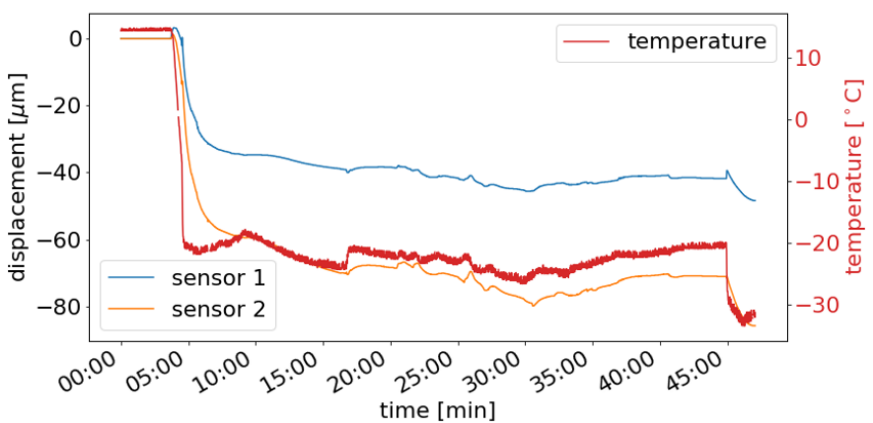

Fig. 9: Measured temperature and displacement of sensors.

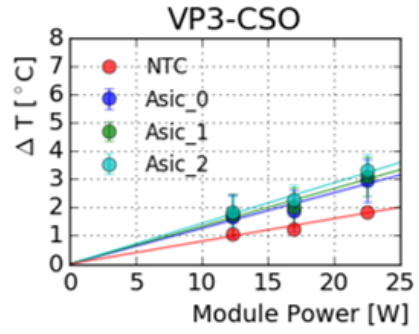

(a) Measured at CERN.

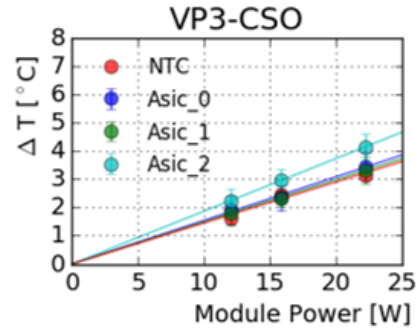

(b) Measured at Manchester.
Fig. 10: Comparison of thermal performance of the same module ASIC at different sites.

thermal shock of a rapid temperature increase is performed as well. A set of communication tests is done before and after cycling to ensure the finished module performs well at all times.

4) Thermal performance: The measurement allows for testing the thermal performance of the module and hence the quality of the glue layer underneath the tiles. The results are projected towards the future increase of power up to $25 \mathrm{~W}$ as the cooling plant is limited by amount of heat it can remove.

The ASIC bandgap values, power consumption and temperatures measured by the NTCs on both front-end hybrids and cooling pipes are recorded. A temperature difference $\Delta T$ between delivered liquid $\mathrm{CO}_{2}$ and ASICs is monitored as a function of tiles power consumption. The dependency for modules measured at CERN and Manchester is in figure 10.

Since the power consumption of the ASICs will change differently according to how close they are to the interaction point, a more complex grading scheme has been developed. The grading is based on $\Delta T$ at $25 \mathrm{~W}$, values set as A ( all ASICs $<5^{\circ} \mathrm{C}$ ), B ( all near beam ASICs $<5^{\circ} \mathrm{C}$ ), C (at most one near beam ASIC $<7^{\circ} \mathrm{C}$ ), $\mathrm{F}$ (any ASIC $>7^{\circ} \mathrm{C}$ ).

\section{Communication test}

1) TFC (timing and fast control): TFC is the LHCbwide synchronous control system, providing information every proton bunch crossing what is to be done. The system can tell the front-end to send out a predefined-pattern sync frames, which are detected in the back-end and used to estimate the latency thus synchronise all links of each subdetector, and

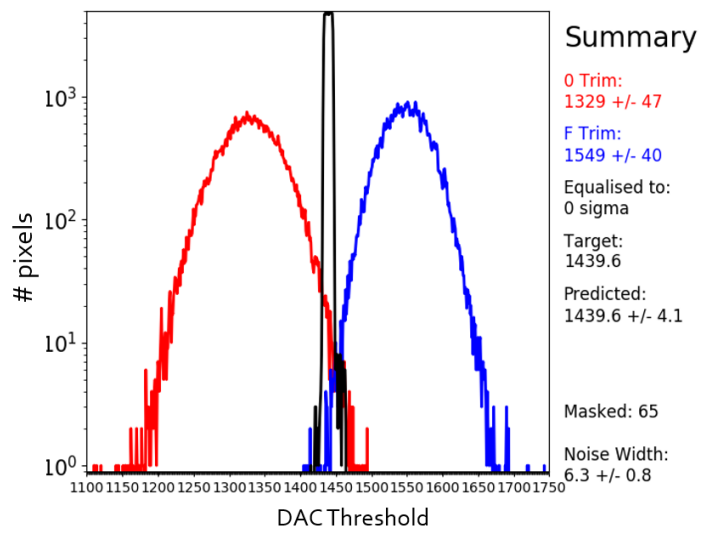

(a) Histogram of equalization result for a single ASIC. Red curve indicates $0 \mathrm{x} 0$ trim setting, blue $0 \mathrm{xF}$ setting as a dependency of noisy pixels for a given threshold. Black curve corresponds to the equalized ASIC.

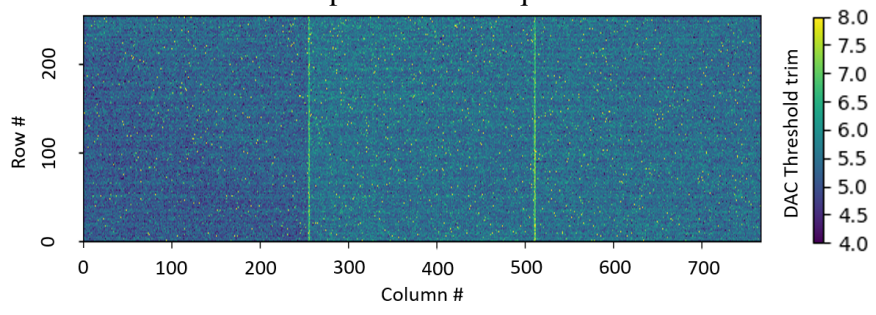

(b) Map of equalized bits for a single tile, color scale corresponds to the individual pixel trim value.

Fig. 11: Equalization results.

also all of the subdetectors together. Other commands include vetos, synchronous rests and more.

The performed tests check the response of the system to different commands, making sure the the communication between frond-end and back-end is as designed.

2) Equalization: In order to obtain consistent response to the signal in every pixel, the individual pixel threshold has to be adjusted. For this purpose the internal logic contains a 4 bit trim DAC (each threshold DAC corresponding to the signal of $10 \mathrm{e}^{-}$) [6], the whole ASIC response is equalized using the per-pixel trim setting. Another important aspect of the equalization is to recognize and mask any noisy or bad pixels, suppressing false signal and flooding of the readout.

The results are in figure 11, visualizing both process of equalization and final trim DAC mapping to the tile.

3) PRBS (pseudo-random bit stream): The operation of the whole VELO requires that data are successfully transferred without any significant deterioration. To ensure good connections and pull phase settings, a PRBS test is performed, checking that the BER (bit error rate) is less than a defined value in all of the 20 links used to read out the data. For this purpose a stream of predefined numbers is generated in each ASIC and sent to the DAQ, where the received bits are compared and error rate is estimated, results shown in figure 12.

Grading of the test is defined using the BER values, results 

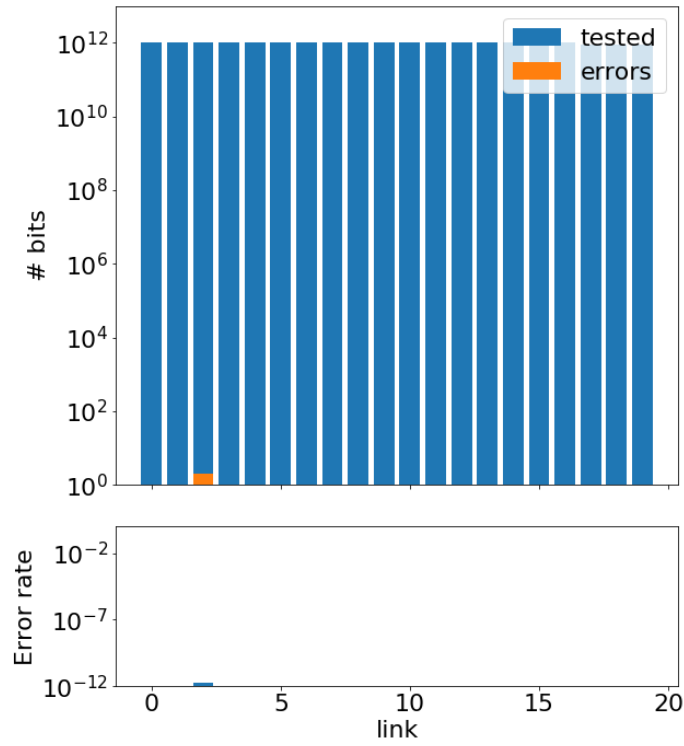

Fig. 12: Results of the PRBS test.

being A (all links $<10^{-12}$ ), B (any links on a different readout tape $>10^{-12}$ ), $\mathrm{C}$ (any links on the same readout tape $>10^{-12}$ ) and $\mathrm{F}$ (any links $>10^{-10}$ ).

\section{CONCLUSION}

As the precision requirements for the VELO upgrade modules are high, different techniques were developed in order to achieve the best quality and longevity of the modules. The information about every production step and state of each module is uploaded to a dedicated online database, where scripts analyze data for further automatic grading.

After the module production is finished and the whole VELO is assembled in Liverpool in the first half of 2020, work for the installation and commissioning of the whole system will start at CERN. The system should be ready for the datataking in the early 2021.

\section{REFERENCES}

[1] A. A. Alves et al., "The LHCb detector at the LHC," Journal of Instrumentation, vol. 3, no. 08, pp. S08 005-S08 005, aug 2008. [Online]. Available: https://doi.org/10.1088\%2F1748-0221\%2F3\% 2F08\%2Fs08005

[2] The LHCb Collaboration, "LHCb VELO Upgrade Technical Design Report," LHCb, Tech. Rep., 2013, CERN-LHCC-2013-021, LHCb-TDR013. [Online]. Available: https://cds.cern.ch/record/1624070

[3] The LHCb Collaboration, "LHCb VELO (VErtex LOcator): Technical Design Report," LHCb, Tech. Rep., 2001, CERN-LHCC-2001-0011, LHCb-TDR-5. [Online]. Available: https://cds.cern.ch/record/504321

[4] R. Aaij et al., "Performance of the LHCb vertex locator," Journal of Instrumentation, vol. 9, no. 09, pp. P09007-P09 007, sep 2014. [Online]. Available: https://doi.org/10.1088\%2F1748-0221\% 2F9\%2F09\%2Fp09007

[5] S. Eisenhardt, "The LHCb upgrade," Journal of Physics: Conference Series, vol. 447, p. 012046, jul 2013. [Online]. Available: https: //doi.org/10.1088\%2F1742-6596\%2F447\%2F1\%2F012046
[6] T. Poikela, M. D. Gaspari, J. Plosila, T. Westerlund, R. Ballabriga, J. Buytaert, M. Campbell, X. Llopart, K. Wyllie, V. Gromov, M. van Beuzekom, and V. Zivkovic, "VeloPix: the pixel ASIC for the LHCb upgrade," Journal of Instrumentation, vol. 10, no. 01, pp. C01 057-C01 057, jan 2015. [Online]. Available: https://doi.org/10. $1088 \% 2 \mathrm{~F} 1748-0221 \% 2 \mathrm{~F} 10 \% 2 \mathrm{~F} 01 \% 2 \mathrm{Fc} 01057$

[7] O. de Aguiar Francisco, J. Buytaert, P. Collins, R. Dumps, M. John, A. Mapelli, and G. Romagnoli, "Evaporative $\mathrm{CO} 2$ microchannel cooling for the LHCb VELO pixel upgrade," Journal of Instrumentation, vol. 10, no. 05, pp. C05014-C05014, may 2015. [Online]. Available: https://doi.org/10.1088\%2F1748-0221\%2F10\%2F05\%2Fc05014

[8] S. de Capua, J. Freestone, S. Klaver, C. Parkes, P. Rodriguez, A. Shtipliyski, and G. Stelmasiak, "Shear and thermal testing of adhesives for VELO upgrade," $L H C b$ PUB, vol. 26, jan 2016. [Online]. Available: https://cds.cern.ch/record/2229009/files/LHCb-PUB-2016-026.pdf 